\title{
Vibration-assisted tunneling: a semiclassical instanton approach
}

\author{
Michele Ceotto \\ Dipartimento di Chimica Fisica ed Elettrochimica, \\ Università degli Studi di Milano, via Golgi 19, 20133 Milano, Italy*
}

\begin{abstract}
A simple method to find the instanton trajectories is developed. This method does not employ any approximation and "exact" instanton trajectories have been located for several collinear symmetric reactions. Underlying the method is the notion of stability of periodic trajectories and the behavior of the action derivatives. Applications to thermal rate constants calculations are presented showing that the method is suitable for quantitative rate constant predictions. In deep tunneling regime, where a classical rate underestimation is about two orders of magnitude, the semiclassical instanton rate is within few percents the exact quantum mechanical value. Then, the consistent amount of tunneling involved in heavy particles transfer, as for the collinear $\mathrm{H}+\mathrm{BrH}$ reaction, shows that "corner-cutting" is not necessary for tunneling to occur, even if it is a sufficient condition to detect a significant presence of tunneling effects.
\end{abstract}

Keywords: instanton, rate constant, collinear reactions, semiclassical, stability matrix, imaginary time

*Electronic address: michele.ceotto@unimi.it 


\section{INTRODUCTION}

Quantum mechanical rate constant theory is the topic of a considerable number of studies, due to the vast experimental evidences that tunneling plays a key role not only in proton transfer, but also in heavier atoms transfer at low temperatures [1, 2]. Quantum thermal rate constant formulation has also been central in W. H. Miller's research. Back in the late sixties and early seventies, Miller showed in a series of papers how to represent quantum observables, such as tunneling, in terms of classical variables [3-7]. These results were welcomed with great interest by the chemical dynamics community because in these papers counter-intuitive quantum phenomena were explained with a coherent and self-consistent classical picture. First, he showed that classical dynamics on the inverted potential is a path approximation for the Boltzmann density matrix $[3,8$, 9]. This is equivalent to the celebrated "Euclidean action", later called instanton action $[10,11]$. Then, he formulated an exact quantum mechanical theory for the thermal rate constant calculations [12]. The semiclassical (Wigner distribution) picture of this formulation and related stationary phase approximations brought him to derive the instanton theory for the rate constant calculation, where only a single trajectory is needed to reproduce tunneling effects [13].

Actually, tunneling was already represented at that time by analytical continuation of the time evolution [14]. Also, Langer et al. [15, 16] derived the metastable decaying time by analytical continuation and, successively, their derivation went under the name of "Im F" [17]. Only later, in the mid-seventies, Coleman [18] and the physics community [10, 11, 19] employed it in theoretical particle physics and called it "the instanton theory". Some distinguo should be made between these theories, where the minima of a double-well potential are assumed to be the instanton turning points and the period is stretched to infinity [20], and those instanton trajectories with finite imaginary-time for thermal rate constant calculations. The instanton theory is still nowadays used by this community with proficiency [21-25].

When the instanton theory for thermal rate constant calculations has been developed by Miller, applications [26] revealed how difficult it was to find instanton trajectories. For these reasons a number of recipes to approximate such trajectories has been proposed. The "t-curve", an instanton path approximation where the vibrational coordinates were fixed, describes tunneling mainly along the reaction coordinate and it is not unique [27]. Later, Truhlar and coworkers [28], developed a series of methods for calculating thermal rate constants. These are approximations of the original instanton method, but easier to implement for complex reactions. One is the zero-curvature tunnel- 
ing (ZCT) approximation [29] and another the small-curvature tunneling (SCT) (or "adiabatic" or "slow-flip") one, in which tunneling trajectory is on the top of the minimum energy path. [30] Beside, there is the large-curvature ground-state tunneling (LCT) method [31], which is also known under various names such as "direct path approximation", "sudden" or "frozen bath" and "fast flip" approximation and it fits the extreme case when the reactive trajectory cuts the corner between the reactants and the products valleys. The SCT and LCT transmission coefficients cover the whole range of reaction-path curvatures, so it was natural to develop a transmission coefficient which was equal to the largest between the SCT and LCT tunneling probabilities. This last approximation is called microcanonical optimized multidimensional tunneling ( $\mu \mathrm{OMT}$ ) method.[32] Finally, the least-action ground-state tunneling (LAT) [33] was developed as a more precise method. These approximations are usually introduced on rather intuitive grounds and judged by comparison with quantum mechanical computations, when the latter are available. However, when tunneling from the ground state is involved rather than small tunneling corrections next to the barrier top, the reaction probability is very sensitive to the choice of the path, and neither one of the above approximations can quantitatively describe the rate constant. To find the exact instanton trajectory and include the correct prefactor, going beyond its frequent unitary approximation, is needed in these cases.

The difficulties in finding the instanton trajectory in many dimension can be bypassed by continuously deforming the potential by changing its parameters. Starting from the situation where the reactive coordinate is uncoupled and the instanton solution is one dimensional, the parameters are gradually changed up to the desired potential $[1,34]$. However this method can not be a priori applied to any potential. Alternatively, correction terms are added to the one-dimensional picture by introducing coupling to the other vibrational degrees of freedom (transverse normal modes) in harmonic approximation [35].

The instanton idea was also at the origin of several quantum mechanical theories formulations or interpretations [36]. For example, the Ring-Polymer Molecular Dynamics has been refined using the instanton periodic trajectories [37]. The instanton idea was also employed for accurate quantum transition state theories formulations, such as the quantum instanton one [38-45].

In this paper, I recall the original Miller's derivation of the semiclassical instanton method and propose a way to find the instanton trajectories without invoking any sort of approximation for the instanton path. Previous "exact" instanton calculations have been limited to the collinear $\mathrm{H}+\mathrm{H}_{2}$ reaction [26]. Here, a different approach than the one used in the past is introduced and 
employed, i.e. inspecting the classical action behavior at different trajectory initial conditions. This method allowed me to graphically determine, within several digits of precision, the initial conditions of the instanton trajectory. After locating such a trajectory, the quantities needed for the rate calculation can be easily obtained. In this way, instanton trajectories for a complete set of symmetric collinear reactions are calculated and the "exact" (without any approximation for the prefactor terms) instanton rate expression is employed in the rate calculation.

This paper is organized as follows. In Section 2 a derivation of the instanton rate in the energy domain is shown and Miller's original derivation is recalled. In Section 3 a criterion for the multidimensional instanton path search is put forward and applied to the symmetric collinear reaction rate calculations shown in Section 4. Section 5 discusses the results and Section 6 concludes the paper.

\section{RECALLING THE SEMICLASSICAL INSTANTON APPROXIMATION}

Instanton dynamics was derived by Miller [13] to calculate thermal rate constants in tunneling regime. A possible quantum formulation of the rate constant is given in terms of the thermal Boltzmann average of the cumulative reaction probability $N(E)$ at energy $E$

$$
k(T)=\frac{1}{2 \pi \hbar Q_{r}(T)} \int_{0}^{\infty} N(E) \exp \left[-E / k_{B} T\right] d E
$$

where $k_{B}$ is the Boltzmann constant and $Q_{r}(T)$ is the reactants partition function.

In a tunneling regime, i.e. for a classical forbidden process, the energy is such that $E<V_{\circ}$, where $V_{\circ}$ is the potential at the top of the barrier. The well-known Jeffreys-Wenzel-KramersBrillouin (JWKB) approximation [46, 47] offers a possible formulation of a quantum probability transition in terms of classical variables [14],

$$
N(E)=\exp \left[-\frac{2 \theta(E)}{\hbar}\right]
$$

where the exponent is

$$
\theta(E)=\operatorname{Im} \int_{\mathbf{q}_{1}}^{\mathbf{q}_{2}} d \mathbf{q}(2 m[E-V(\mathbf{q})])^{1 / 2}
$$

$T$ is the trajectory total time at energy $E, \mathbf{q}_{1}$ and $\mathbf{q}_{2}$ are the multidimensional turning points and they delimit the tunneling region or what is also called the "interaction region" $[1,13]$. 
After substituting Equations (II.2) and (II.3) into the rate expression Equation (II.1), the integration in $E$ is done by steepest descent approximation. The stationary energy point $E=E(\beta)$ is given by inverting the following equation [4]

$$
\begin{aligned}
\hbar \beta(E) & =-2 \frac{d \boldsymbol{\theta}(E)}{d E} \\
& =2 \int_{\mathbf{q}_{1}}^{\mathbf{q}_{2}} d \mathbf{q}\left(\frac{m}{2[V(\mathbf{q})-E]}\right)^{1 / 2} \equiv \tau(E)
\end{aligned}
$$

which determines the period $\tau(E)$ of the instanton trajectory. Equation (II.4) is pivot in instanton theory: It relates the temperature with the imaginary time period of the instanton trajectory, no matter what shape the potential barrier is. In order to complete the steepest descent integration the second order derivative is calculated

$$
-2 \frac{d^{2} \theta(E)}{d E^{2}}=\frac{d \tau(E)}{d E}=\frac{\hbar}{E^{\prime}(\beta)}
$$

where the equivalence in Equation (II.4) between $\beta$ and the imaginary time is used. Another way to derive Equation (II.4) is shown in Appendix A. In conclusion, the rate of Equation (II.1) becomes

$$
k(T)=\frac{1}{Q_{r}(T)} \frac{\sqrt{-2 \pi E^{\prime}(\beta)}}{2 \pi \hbar} \exp \left[-\beta E(\beta)-\frac{2 \theta(E(\beta))}{\hbar}\right]
$$

or by writing the prefactor in terms of $\theta(E)$ is

$$
\begin{aligned}
k(T) & =\frac{1}{Q_{r}(T)} \frac{1}{2 \pi \hbar} \sqrt{\frac{2 \pi \hbar}{2 \theta^{\prime \prime}(E(\beta))}} \\
& \times \exp \left[-\beta E(\beta)-\frac{2 \theta(E(\beta))}{\hbar}\right]
\end{aligned}
$$

This is the JWKB results for thermal rate in classical forbidden regime and it shows how imaginary time and periodic motion are able to describe the thermal rate. In Equation (II.3) the integral depends both on the barrier height and width. Therefore, the reacting particle has to choose between the short way with a high barrier and the long passage with a low barrier. The actual tunneling path is a compromise between these two factors.

As mentioned in the Introduction, Miller derived a fully consistent semiclassical expression of the rate in tunneling regime. The idea was strictly related to the original Transition State Theory (TST) one. Miller's derivation is recalled in Appendix B and the reader should refer to his original papers for details $[4,13]$. His final result is very similar to Equation (II.7) and it resembles the TST one, 


$$
\begin{aligned}
k(T) & =\frac{1}{Q_{r}(T)} \frac{1}{2 \pi \hbar} \sqrt{-2 \pi E^{\prime}(\beta)} e^{-\phi(E) / \hbar} \\
& \times \prod_{i=1}^{F-1} \frac{1}{2 \sinh \left[u_{i}(E) / 2\right]}
\end{aligned}
$$

where $i=1, \ldots, F-1$ are the degrees of freedom perpendicular to the reaction coordinate, $u_{i}(E)$ the stability parameters [13] and

$$
\begin{aligned}
\phi(E) & =\hbar \beta E-i \int_{0}^{T(E)} d t \mathbf{p}(t) \dot{\mathbf{q}}(t) \\
& =\int_{0}^{\hbar \beta} d \tau H(\overline{\mathbf{p}}(\tau), \mathbf{q}(\tau))
\end{aligned}
$$

is the action of trajectories with energy $E=-\overline{\mathbf{p}}(\tau)^{2} / 2 m+V(\mathbf{q}(\tau))$ and $\tau=i t$ is the analytical continuation of the real time evolution. Equation (II.8) is the working expression of this paper for the rate constants calculation. The integral $\int_{0}^{T(E)} d t \mathbf{p}(t) \dot{\mathbf{q}}(t)$ is also called "least action" $S_{L A}$. The action and the energy in Equation (II.8) are related as in (real-time) classical mechanics

$$
\begin{aligned}
\frac{d}{d \beta} \phi(E) / \hbar & =\frac{d}{d \beta}\left[\beta E(\beta)-\frac{i}{\hbar} \int_{0}^{T(E)} d t \mathbf{p}(t) \dot{\mathbf{q}}(t)\right] \\
& =E(\beta)+\left[\beta-\frac{i}{\hbar} \frac{d \int_{0}^{T(E)} d t \mathbf{p}(t) \dot{\mathbf{q}}(t)}{d E}\right] E^{\prime}(\beta) \\
& =E(\beta)
\end{aligned}
$$

where Equation (VI.13) was employed. $E(\beta)$ in Equation (II.8) is the equivalent of the activation energy in TST. In fact, the whole Equation (II.8) exhibits an interesting resemble to conventional TST. When the stability parameters are $u_{i}(E(\beta))=\hbar \omega_{i} \beta$ in harmonic approximation, where $\left\{\omega_{i}\right\}$ are the vibrational frequencies of the transition state, then the product $\prod_{i=1}^{F-1}$ gives the partition function of the activated complex in harmonic approximation.

The analytical continuation introduced naturally by the stationary phase approximation of Equation (VI.12) has an interesting physical interpretation [3, 48]. After applying the Wick's rotation to the classical equation of motion [3]

$$
\begin{aligned}
t & =-i \tau \\
m \ddot{x}(\tau) & =+\frac{d V(x(\tau))}{d x}
\end{aligned}
$$

the instanton dynamics becomes equivalent to performing classical mechanics on the inverted potential. The solution of Equation (II.12) are the instanton trajectories and their momenta are

$$
\bar{p} \equiv m(d x / d \tau)=-i m(d x / d t)=-i p
$$


and the instanton action

$$
\begin{array}{r}
\phi\left(x_{1}(0), x_{2}(\tau), E\right)= \\
\int_{0}^{\hbar \beta} d \tau\left[\frac{1}{2} \bar{p}(\tau) q \dot{(\tau)}+V(q(\tau))\right]
\end{array}
$$

where $x_{1}(0)$ and $x_{2}(\tau)$ are the turning points. In particular, $x_{1}(0)$ is the inversion point for a real-time classical trajectory impinging the barrier from the reactants and $x_{2}(\tau)$ from the products. The reader will note that the integrand of Equation (II.14) is the Hamiltonian in terms of the pure imaginary time and the instanton action becomes

$$
\phi(E)=\int_{0}^{\hbar \beta} d \tau H(\bar{p}(\tau), q(\tau))
$$

which is also called "Euclidean action" [10,21].

\section{A CRITERION FOR THE MULTIDIMENSIONAL SEARCH OF INSTANTON TRAJECTO- RIES}

As mentioned in the Introduction, a major obstacle to the instanton application is the determination of the trajectory. In this section a criterion for determining the instanton trajectory is derived. This method allows me to test several combinations of symmetric collinear reactions.

Search of instanton trajectories is far from trivial in many dimensions. Hereafter some general considerations are given in order to develop a method for finding multidimensional instanton periodic trajectories. Let's consider a bi-dimensional system, such as a collinear reaction. At any time $t$, the trajectory is located in coordinates space at $\mathbf{q}(t)=\left(q_{1}(t), q_{2}(t)\right)$ and the total energy is

$$
E=\frac{1}{2}\left(\dot{q}_{1}^{2}+\dot{q}_{2}^{2}\right)+V\left(q_{1}, q_{2}\right)
$$

where the mass is unitary and $V\left(q_{1}, q_{2}\right)$ is the potential energy. Four arbitrary constants are involved in the solution of these differential equations of motion. Dropping the constant additive to $t$, the epoch in the orbit, one is left with $\infty^{3}$ distinct orbits. There are a number of $\infty^{2}$ of these orbits that share the same total energy $E$. Then, this set of periodic orbits can be further divided into "ordinary" and "singular" ones. In the first case, it is possible to find other periodic orbits with the same energy $E$ that can be transformed into the original orbit by infinitesimal transformations. In the second case, there are no such orbits. For the collinear set of reactions considered in this 
paper, the instanton trajectories are bi-dimensional. It has been proved that all periodic trajectories in two dimensions are singular ones [49].

In order to locate the instanton trajectories, I will employ the classical action's properties for periodic trajectories. Since Newton dynamics is performed, the Hamiltonian action on the inverted potential depends on the initial positions and momenta, and on the total time. Total time is fixed to be equal to $\hbar \beta$ and the action is

$$
\phi\left(\mathbf{q}^{\prime}, \mathbf{p}^{\prime}, \hbar \beta\right)=\int_{0}^{\hbar \beta}\left(\mathbf{p}\left(\mathbf{q}^{\prime}, \mathbf{p}^{\prime}, t\right) \dot{\mathbf{q}}\left(\mathbf{q}^{\prime}, \mathbf{p}^{\prime}, t\right)-H\left(\mathbf{p}\left(\mathbf{q}^{\prime}, \mathbf{p}^{\prime}, t\right) \mathbf{q}\left(\mathbf{q}^{\prime}, \mathbf{p}^{\prime}, t\right)\right)\right) .
$$

By taking the first derivative of Equation (III.2) respect to the initial position with fixed initial momenta and time, and by using Hamilton equations, I obtain

$$
\left.\frac{\partial \phi}{\partial \mathbf{q}^{\prime}}\right|_{\mathbf{p}^{\prime}, \hbar \beta}=\mathbf{p}(\hbar \beta) \frac{\partial \mathbf{q}(\hbar \beta)}{\partial \mathbf{q}^{\prime}}-\mathbf{p}^{\prime} .
$$

Equation (III.3) is in general different from zero, except for a trajectory of period $\hbar \beta$, i.e. the instanton one. I will use this property to locate the instanton trajectories at different temperatures. I would expect also to find only one initial set of conditions for the first derivative to be zero, because in two dimensions instantons trajectories are singular ones.[49]

The mass-scaled normal modes coordinates are adopted. For the following collinear reactions, $\mathrm{H}+\mathrm{H}_{2} ; \mathrm{H}+\mathrm{BrH} ; \mathrm{Cl}+\mathrm{HCl} ; \mathrm{Cl}+\mathrm{DCl}$, the PotLib database is employed, [50] and the surface dividing the reactants from the products is given by the symmetric stretch coordinate subspace, i.e. any asymmetric stretch displacement moves the system away from the dividing surface. This is the dashed vertical line in Figure 1 and the transition state is located at the cross placed on the same Figure. [Place Figure 1 here] Thus, any asymmetric stretch will bring the transition state either into the products or the reactants valley and the instanton trajectories will cross the dividing surface perpendicularly. Accordingly to Equation (III.3), the search for the singular periodic instanton trajectories is conducted by starting classical trajectories at the dividing surface with momentum perpendicular to the surface itself on the inverted potential. This choice guarantees that after half period the trajectory will have the same momentum but with opposite direction, as requested by the symmetry of the potential. The momentum fixes the total energy of the trajectory and ultimately the temperature for the rate constant calculation. Initially, a grid of very dense initial points was set along the dividing surface and trajectories were run starting from these points with the same momentum. None of these trajectories turned out to be periodic, since the grid was not dense enough. To better explain how dense this grid should be in order to find the instanton trajectory, 
in Figure 1 three trajectories with the same momenta and starting from very close initial positions at the dividing surface are reported. It is not possible to distinguish by naked eye each path up to the turning point. Nevertheless, at the turning points the three trajectories behave dramatically differently and only the red one is periodic. This strong dependency on the initial conditions, which is the hallmark of chaotic systems, makes the search for instanton trajectories by looking at their initial conditions an extremely unstable numerical problem. Moreover, there is no idea a priori where the instanton trajectory maybe even roughly located, if not using Equation (III.3). Hence, it is necessary to elaborate a different approach for finding instanton trajectories than simply setting up a grid of initial conditions.

As explained above, trajectories are tested by varying only one parameter $q_{2}$, which is the initial position along the dividing surface, while $q_{1}$ is kept fixed at the dividing surface location. Momentum component $p_{1}$ perpendicular to the dividing surface is the only component to be different from zero and it is fixed at each temperature. The variations of the trajectory actions and derivatives with respect to this parameter are reported in Figure 2.[Place Figure 2 here] The initial conditions of the periodic trajectory represented in red line and arrows in Figure 2 is determined by the action first derivative (middle panel of Figure 2) which is equal to zero when the trajectory is periodic, or by the change of slope of the action itself (upper panel in Figure 2). This last consideration is true both for the Hamilton action and the least action. Since the discontinuity of the first derivative occurs in a lambda-type fashion, there are formal similarities with the specific heat behavior during a second order phase transition. The second derivative stresses this behavior by tending to infinity. These plots and considerations allow for graphical determination of the initial conditions of the instanton trajectory. Subsequently these can be refined up to several digits to obtain periodic trajectories. This search has been conducted in one dimension, i.e. by reducing the set of initial conditions to a single one. However, the method can be extended in two dimensions. By varying the initial momentum perpendicular to the dividing surface $p_{1}(0)$ versus the position along the surface $q_{2}(0)$, instanton trajectories are graphically determined by plotting the stability matrix eigenvalues as in Figure 3 [Place Figure 3 here]. The set of initial conditions for periodic trajectories is given by the combination of $p_{1}(0)$ and $q_{2}(0)$ along the line marked by the eigenvalues. At any given temperature, it is possible to calculate the corresponding energy and the initial momentum $p_{1}(0)$, and locate the initial position $q_{2}(0)$ from a plot of the type of Figure 3. A possible procedure in many dimensions is first to locate the transition state, then to test trajectories of a small time period starting from several points on the dividing surface and surrounding 
the transition state point. The instanton trajectory will be located by inspecting the action first or second derivative for these starting points. [Place Figure 4 here] It is also quite instructive to look at the instanton trajectories phase space plots reported in Figure 4: On one hand this plot strictly checks the accuracy of the orbits after the trajectory is run for several periods; on the other hand, it is interesting to note that on the reactive (asymmetric) sub-phase-space plot the trajectory exhibits a kind of cusp at the turning points. This is the source of the chaotic behavior reported above and it is amplified at low temperatures because of the longer time period.

Following this procedure, the instanton trajectories of four types of collinear reactions at several temperatures were found and plotted in Jacobi's coordinates in Figure 5. [Place Figure 5 here] The reactions were chosen in order to test the ability of the instanton theory to calculate the rate constants of the light-heavy-light combination $(\mathrm{H}+\mathrm{BrH})$, the light-light-light combination $(\mathrm{H}+$ $\left.\mathrm{H}_{2}\right)$, the heavy-light-heavy combination $(\mathrm{Cl}+\mathrm{HCl})$ and isotope effects $(\mathrm{Cl}+\mathrm{DCl})$. The skew Jacobi angle is representative of the different masses combination and it ranges from about $13^{\circ}$ for $\mathrm{Cl}+\mathrm{HCl}$, to exactly $60^{\circ}$ for $\mathrm{H}+\mathrm{H}_{2}$ and almost $90^{\circ}$ for $\mathrm{H}+\mathrm{BrH}$. A common feature of all instanton trajectories in Figure 5 is that for smaller $\hbar \beta$ periods, i.e. higher temperatures, the initial momentum gradually goes to zero and the initial position shifts towards the Transition State (TS) location. Eventually, when the total energy $E$ becomes equal to the potential energy of the TS, the initial momentum is $\mathbf{p}(0)=0$ and the infinitesimal instanton trajectory is the TS point. The cross-over temperature is $T_{C}=\hbar \omega^{\ddagger} / 2 \pi k_{B}$, where $\omega^{\ddagger}$ is the frequency of the inverted potential along the reactive coordinate at the TS geometry. For $T>T_{C}$, the instanton trajectory exhibits imaginary turning points. Another common feature regards the instanton turning points: These are located along the minimum energy path (MEP), as shown in Figure 5 [51]. During a wavepacket propagation simulation, the packet would be found traveling and centered on the top of the MEP and to be reflected or transmitted at these points. A light-heavy-light combination (such as $\mathrm{H}+\mathrm{BrH}$ ) where a heavy mass is transferred presents an instanton path which quite closely reflects the MEP shape. Instead, when a heavy-light-heavy reaction occurs (such as $\mathrm{Cl}+\mathrm{HCl}$ ) and a light mass is transferred, the instanton trajectory departs from the classical MEP profile. In this case the instanton trajectory resembles a shortcut from products to reactants by "cutting the corner".[52-55] An impressive amount of energy should be spent to climb across the barrier along this classical path. Instead, tunneling effects open this route "for free". A less significant "cornercutting" occurs for a light-light-light $\left(\mathrm{H}+\mathrm{H}_{2}\right)$ collinear reaction. Nevertheless, tunneling is severe in this case as well, and it persists above room temperature $(\mathrm{T}=350 \mathrm{~K})$. In general, the smaller the 
skew angle is, the stronger is the coupling between the modes. Finally, an isotopic substitution is presented in the panel $(\mathrm{Cl}+\mathrm{DCl})$. Here, at any temperature the arc length between the instanton trajectory turning points is much shorter than in the hydrogen transfer case and it can be deduced that tunneling is significantly less deeper than the $\mathrm{Cl}+\mathrm{HCl}$ reaction.

[Place Figure 6 here ] In Figure 6 the trajectories of Figure 5 are reported in bond coordinates (the two distances between the three atoms on a line) reference system. During the transfer of a Bromine atom, synergistic variations of both coordinates occur. Instead, in the heavy-light-heavy cases $(\mathrm{Cl}+\mathrm{HCl}$ and $\mathrm{Cl}+\mathrm{DCl})$ the heavy atoms are stopped while the light one is transferred. In those cases, the instanton trajectory is well described by the equation $R_{1}+R_{2}=C$, where $C$ is a constant and $R_{i}$ is a bond coordinate. With an isotopic substitution, tunneling effects are remarkably quenched: For the $\mathrm{Cl}+\mathrm{DCl}$ reaction, the critical temperature $\mathrm{T}_{C}$ is already reached at $\mathrm{T}=250 \mathrm{~K}$. Question remains whether one can estimate how much deep the tunneling is for each one of these reactions. [Place Figure 7 here] In Figure 7 the variation of the potential $V(\mathbf{q}(\tau))$ along the instanton orbit is reported for half period trajectory at $T=150 \mathrm{~K}$. The zero potential value corresponds to the reactants and products geometry for each reaction. Thus, the tunneling potential profile for the $\mathrm{Cl}+\mathrm{HCl}$ and $\mathrm{Cl}+\mathrm{DCl}$ can be directly compared and it can be deduced that tunneling is deeper for the first case because the variation of the potential during the instanton excursion is about two times the isotopic reaction one. Comparable potential variation is experienced for the $\mathrm{H}+\mathrm{BrH}$ reaction, while this is only about half of that one occurring during the $\mathrm{H}+\mathrm{H}_{2}$ reaction. Two important observations are possible based on these results. First, deep tunneling can occur without having a "corner-cutting" trajectory. Second, an instanton path that resembles the MEP is not necessarily including less tunneling amount than a corner-cutting path.

\section{THERMAL RATE CONSTANTS CALCULATIONS}

The thermal rate constants are calculated accordingly to Equation (II.8). After finding the instanton trajectory, the stability parameters $u_{i}(E)$ are obtained by diagonalizing the stability matrix after one period of evolution and the instanton action $\phi(E)$ according to Equation (II.9). The first derivate of the energy $E(\beta)$ is obtained in two steps. First the value of $E(\beta)$ is calculated for several temperatures within the range of interest. [Place Figure 8 here] Then, the first derivative of $E(\beta)$ is calculated from the fitted curves. Figure 8 shows that the value of $E(\beta)$ and of its variation is smooth for all collinear reactions. In Figure 8, tunneling effects persist at room 
temperatures (at $\mathrm{T}=300 \mathrm{~K}, \beta=1052$ a.u.) for the collinear reaction $\mathrm{H}+\mathrm{H}_{2}$, while they are important mainly at low temperatures for the other reactions. In the same Figure, the isotope effects can be appreciated by comparing the green $\mathrm{Cl}+\mathrm{HCl}$ energy profile with the cyan one for the $\mathrm{Cl}+\mathrm{DCl}$ reaction. The isotopic substitution quenches significantly the contribution of tunneling, considering that the rate is exponentially energy dependent in a TS model. Both a cubic polynomial and Chebyshev-series representation was employed to generate the spline equation.[56] The Chebyshev-series representation turned out to be more reliable, since it avoids spurious oscillations. In the Chebyshev approach, the variable is normalized, i.e. scaled to an interval $[-1,1]$ and the Chebyshev coefficients are calculated. Then, a polynomial is extracted from the set of coefficients. This procedure turned out to reduce rounding errors and to be numerically stable, i.e. to converge for several different collections of energy data. This fitting procedure was applied for interpolations (and not for extrapolations) of the values of $E(\beta)$ and an order equal to 4 was accurate enough not to change the value of the rates within several digits if higher order splines would have been employed. Finally $E^{\prime}(\beta)$ was calculated by finite differences.

The thermal rate constants calculated by Equation (II.8) are reported in Figure 9. [Place Figure 9 here] On each panel the black squares are the exact quantum mechanical results and the cyan diamonds the classical TST ones [57-59]. On the same panels the red circles are the results of the semiclassical instanton trajectories from Equation (II.8). On the upper left panel, the rates of the $\mathrm{H}+\mathrm{BrH}$ are reported. The contribution of tunneling is by far more important than in other collinear systems and the failure of the TST is striking. Instead, the instanton theory reproduces quite well the exact rate, even at very low temperatures where the discrepancy between classical and quantum rate is about two orders of magnitude. These results are remarkable since obtained with a single classical trajectory, while other real-time semiclassical simulations usually employ many thousands of trajectories when tunneling is so severe. The instanton results are quite accurate for the $\mathrm{H}+\mathrm{H}_{2}$ rates in the upper right panel of Figure 9 as well. In this case the instanton trajectory search was limited at $\mathrm{T}=200 \mathrm{~K}$ because the chaotic nature of the potential was greatly enhanced at low temperatures (long simulation times). In the case of a heavy-light-heavy combination (lower panels of the same Figure) the semiclassical instanton results are somehow more accurate for the $\mathrm{Cl}+\mathrm{DCl}$ than the $\mathrm{Cl}+\mathrm{HCl}$ one. The overestimate of the classical TST at high temperatures is due to the huge recrossing effects occurring for this reaction at these temperatures.

Overall this set of simulations assess the ability of the semiclassical instanton to predict the reaction rate constants even in deep tunneling regime. 


\section{DISCUSSIONS}

During the past thirty years, several ways for finding the instanton trajectories have been used. Nevertheless, the accuracy of these methods versus the "exact" instanton one has been rarely tested, because of the difficulties in finding the instanton trajectories. More often approximate instanton methods have been compared with exact quantum mechanical results or not compared at all with any other method. I think that agreement with quantum results can be sometime fortuitous. Only a direct comparison with the "exact" instanton trajectory results can reveal the level of accuracy of an approximate instanton method. The results presented here can offer a common playground where this comparison occurs. Previous results were limited to the collinear $\mathrm{H}+\mathrm{H}_{2}$, while in this paper a set of collinear thermal rate constants for reactions of the type light-heavylight, light-light-light, heavy-light-heavy and isotopic substitution have been calculated.

\section{CONCLUSIONS}

In this paper we have recalled Miller's instanton derivation and instanton thermal rate constant formulation. A way to find instanton trajectories for symmetric collinear reactions has been presented and thermal rates are calculated. Agreement between quantum results and instanton ones is quite impressive, considering that a single classical trajectory is used here. For example, when classical TST underestimates the thermal rate by two orders of magnitude, the instanton rate is within few percents of accuracy. Further investigations for extending the present method for asymmetric reactions should be considered.

\section{Acknowledgments}

This paper is dedicated with great appreciation to Professor W. H. Miller on the occasion of his 70th birthday in recognition of his formative and original mentoring to my $\mathrm{PhD}$ studies. 


\section{Appendix A}

Another way to reach the result in Eq. (II.5) is to start from the Hamilton-Jacobi equation for a classical real time motion where

$$
\frac{\partial S_{t}}{\partial t}+H(\mathbf{p}(t), \mathbf{q}(t))=0
$$

and after deriving each equation side by the imaginary time, one obtains

$$
\frac{\partial^{2} S_{\beta}}{\partial(\hbar \beta / 2)^{2}}+\frac{2}{\hbar} E^{\prime}(\beta)=0
$$

and this can be simplified as follow

$$
\begin{aligned}
\frac{\partial}{\partial(\hbar \beta / 2)}\left(\frac{2}{\hbar} \frac{\partial S}{\partial E} E^{\prime}(\beta)\right)+\frac{2}{\hbar} E^{\prime}(\beta) & = \\
\frac{4}{\hbar^{2}} \frac{\partial}{\partial E} E^{\prime}(\beta)\left(\frac{\partial S}{\partial E} E^{\prime}(\beta)\right)+\frac{2}{\hbar} E^{\prime}(\beta) & = \\
\frac{4}{\hbar^{2}} E^{\prime 2}(\beta) \frac{\partial^{2} S}{\partial E^{2}}+\frac{2}{\hbar} E^{\prime}(\beta) & =0
\end{aligned}
$$

which is equivalent to the equality

$$
\frac{\partial^{2} S}{\partial E^{2}}=-\frac{\hbar}{2 E^{\prime}(\beta)} .
$$

\section{Appendix B}

Here I recall Miller's derivation of the thermal rate constant formula using the instanton approximation.

First, a dividing surface is introduced and a Wigner phase space distribution is adopted for the classical trajectories sampling. Then, the rate is written in the following form

$$
k(T)=\frac{1}{Q_{r}(T)} \int d \mathbf{q} \delta\left(q_{s}\right) \frac{1}{2}\left|\dot{q}_{s}\right|\left\langle\mathbf{q}\left|e^{-\beta \hat{H}}\right| \mathbf{q}\right\rangle,
$$

where the integral is confined to the dividing surface by the Dirac's delta $\delta\left(q_{s}\right)$ and the reactive flux is weighted by the Boltzmann distribution. Then, it is useful to recall the Gutzwiller's trace formula [60], where the element of matrix of the Green's function $G(E)$ are expressed in terms of periodic classical trajectories in a multidimensional phase space $(\mathbf{q}, \mathbf{p})$. In particular, by selecting the 
degree of freedom $q_{s}$ in which periodic orbits are stable and integrating over all possible periodic trajectories, the following relation holds

$$
\begin{array}{r}
\int d q_{2} \int d q_{3} \ldots \int d q_{F}\langle\mathbf{q}|G(E)| \mathbf{q}\rangle= \\
\frac{i}{\hbar} \frac{1}{\left|\dot{q}_{s}\right|} \sum_{k=1}^{\infty}(-1)^{k} e^{i S_{L A}(E) / \hbar} \times \\
\prod_{i=1}^{F-1} \frac{1}{2 \sinh \left[k u_{i}(E) / 2\right]}
\end{array}
$$

where $G(E)=(E-H)^{-1}$, the $k$-sum is over multiple passes of the periodic trajectory, and $\left\{u_{i}\right\}$ ( $i=1, \ldots, F-1$ and $F$ are the number of degrees of freedom) are the stability parameters of the periodic trajectories $[13,61] . S_{L A}(E)$ is the least action calculated in the entire period $T(E)$

$$
\begin{aligned}
S_{L A}(E) & =\int_{0}^{T(E)} d t \mathbf{p}(t) \cdot \dot{\mathbf{q}}(t) \\
& =2 \int_{\mathbf{q}_{1}}^{\mathbf{q}_{2}} d \mathbf{q} \sqrt{2 m(E-V(\mathbf{q}))} .
\end{aligned}
$$

Then, using the relation

$$
e^{-\beta \hat{H}}=\frac{-1}{2 \pi i} \int d E e^{-\beta E} G(E)
$$

Equation (VI.6) becomes

$$
\begin{array}{r}
\int d q_{2} \int d q_{3} \ldots \int d q_{F}\left\langle\mathbf{q}\left|e^{-\beta E}\right| \mathbf{q}\right\rangle= \\
\frac{1}{2 \pi \hbar} \int d E e^{-\beta E} \frac{1}{\left|\dot{q}_{s}\right|} \sum_{k=1}^{\infty}(-1)^{k-1} e^{i k S_{L A}(E) / \hbar} \times \\
\prod_{i=1}^{F-1} \frac{1}{2 \sinh \left[k u_{i}(E) / 2\right]}
\end{array}
$$

After substituting Equation (VI.9) in to Equation (VI.5), the following rate expression was obtained

$$
k(T)=\frac{1}{2 \pi \hbar Q_{r}(T)} \int d E e^{-\beta E} \int d q_{s} \frac{1}{2} \delta\left(q_{s}\right) P(E)
$$

where

$$
P(E)=\sum_{k=1}^{\infty}(-1)^{k-1} e^{i k S_{L A}(E) / \hbar} \prod_{i=1}^{F-1} \frac{1}{2 \sinh \left[k u_{i}(E) / 2\right]}
$$

is the semiclassical cumulative reaction probability for the $k$ periodic trajectories of energy $E$. The integration in $q_{s}$ is easily performed at the dividing surface, and Equation (VI.10) assumes the more familiar expression

$$
k(T)=\frac{1}{2 \pi \hbar Q_{r}(T)} \int d E e^{-\beta E} P(E) .
$$


Once again the energy integration is performed by using the steepest descent and the semiclassical rate expression corresponding to Equation (II.7) is found. Specifically, first $k$ is fixed $(k=1)$ in Equation (VI.11), since the multiple crossings contribution is negligible. Then the stationary points are such that

$$
\hbar \beta=i \frac{d S_{L A}(E)}{d E}=i T(E)=\tau(E)
$$

which is similar to Equation (II.4). By performing the second derivative of the stationary phase, Equation (VI.12) was approximated by

$$
\begin{aligned}
k(T) & =\frac{1}{Q_{r}(T)} \frac{1}{2 \pi \hbar} \sqrt{-2 \pi E^{\prime}(\beta)} e^{-\phi(E) / \hbar} \\
& \times \prod_{i=1}^{F-1} \frac{1}{2 \sinh \left[u_{i}(E) / 2\right]}
\end{aligned}
$$

where $E=E(\beta)$ is determined by the stationary condition in Equation (VI.13) and the products $\prod_{i=1}^{F-1}$ run over all the degrees of freedom except the reactive one.

[1] V.A. Benderskii, D.E. Makarov, and C.A. Wight, Chemical Dynamics at Low Temperatures, Adv. Chem. Phys. LXXXVIII (Wiley, New York, 1994) and references therein.

[2] R.P. Bell, The tunnel Effect in Chemistry, (Chapman \& Hall, London, 1980).

[3] W.H. Miller, J. Chem. Phys. 55 (7), 3146 (1971).

[4] W.H. Miller and T. George, J. Chem. Phys. 56, 5668 (1972).

[5] J. D. Doll, T. F. George, and W. H. Miller, J. Chem. Phys. 58, 1343 (1973).

[6] W. H. Miller, Adv. Chem. Phys. 25, 69 (1974).

[7] W. H. Miller, J. Chem. Phys. 53, 3578 (1970); ibidem 53, 1949 (1970); W. H. Miller, Adv. Chem. Phys. 30, 77 (1975).

[8] S.M. Hornstein and W.H. Miller, Chem. Phys. Lett. 13 (3), 298 (1972).

[9] W. H. Miller, J. Chem. Phys. 58 (4), 1664 (1973).

[10] A.A. Belavin, A.M. Polyakov, A.S. Schwartz, and Yu.S. Typkin, Phys. Lett. 59B, 85 (1975).

[11] N. V. Krasnikov, V. A. Rubakov, and V. F. Tokarev, Phys. Lett. 79B, 423 (1978); N. V. Krasnikov, V. A. Rubakov, and V. F. Tokarev, Soc. J. Nucl. Phys. 29, 581 (1979).

[12] W. H. Miller, J. Chem. Phys. 61 (5), 1823 (1974).

[13] W. H. Miller, J. Chem. Phys. 62 (5), 1899 (1975). 
[14] L.D. Landau and E.M. Lifšits, Quantum Mechanics (Butterworth, Oxford, 1977).

[15] J.S. Langer, Annals of Physics 41, 108 (1967); J.S. Langer, Ann. Physics 54, 258 (1969).

[16] A. Schmid, Ann. Physics 170, 333 (1986).

[17] U. Weiss, H. Grabert, P. Hanggi, and P. Riseborough, Phys. Rev. B 35, 9535 (1987); R. Gorlich and U. Weiss, Phys. Rev. B 38, 5245 (1988).

[18] S. Coleman, Whys of Subnuclear Physics, (Ed. A. Zichichi, Plenum Press, New York) p. 805 (1979); S. Coleman, Phys. Rev. D 15, 2929 (1977).

[19] Auerbach, S. Kivelson, and D. Nicole, Phys. Rev. Lett. 53 (5), 411 (1984).

[20] G. V. Milnikov, K. Yagi, T. Taketsugu, H. Nakamura, and K. Hirao, J. Chem. Phys. 119, 10 (2003); G. V. Milnikov and H. Nakamura, J. Chem. Phys. 117, 10081 (2002); G. V. Milnikov and H. Nakamura, J. Chem. Phys. 115, 6881 (2001); G. Milnikov and H. Nakamura, Phys. Chem. Chem. Phys 10 (10), 1374 (2008).

[21] S. Yu. Khlebnikov, V.A. Rubakov, and P.G. Tinyakov, Nucl. Phys. B 367, 334 (1991); G.F. Bonini, A.G. Cohen, C. Rebbi, and V.A. Rubakov, Phys. Rev. D 60, 076004 (1999).

[22] F. Bezrukov, D. Levkov, C. Rebbi, V. Rubakov, and P. Tinyakov, Phys. Rev. D 68, 036005 (2003).

[23] F. Bezrukov and D. Levkov, Zh. Eksp. Teor. Fiz. 125, 938 (2004) [J. Exp. Theor. Phys. 98, 820 (2004)]

[24] D.G. Levkov, A.G. Panin, and S.M. Sibiryakov, Phys. Rev. Lett. 99, 170407 (2007).

[25] A. Garg, E. Kochetov, K.-S. Park, and M. Stone, J. Math. Phys. 44 (1), 48 (2003).

[26] S. Chaoman, B.C. Garrett, and W.H. Miller, J. Chem. Phys. 63 (56), 2710 (1975).

[27] R. A. Marcus and M. E. Coltrin, J. Chem. Phys. 67, 2609 (1977).

[28] B.C. Garrett, D.G. Truhlar, J. Chem. Phys. 79, 4931 (1983); R. Skodje, D.G. Truhlar, B.C. Garrett, J. Phys. Chem. 85, 3019 (1981); B.C. Garrett, D.G. Truhlar, A.F. Wagner, J.T.H. Dunning, J. Chem. Phys. 78, 4400 (1983); B.C. Garrett, N. Abusalbi, D.J. Kouri, D.G. Truhlar, J. Chem. Phys. 83, 2252 (1985).

[29] D.G. Truhlar and A. Kupperman, J. Am. Chem. Soc. 93, 1840 (1971).

[30] D.-H. Lu, T.N. Truong, V.S. Melissas, G.C. Lynch, Y.-P. Liu, B.C. Garrett, R. Steckler, A.D. Isaacson, S.N. Rai, G.C. Hancock, J.G. Lauderdale, T. Joseph, D.G. Truhlar, Comput. Phys. Commun. 71, 235 (1992); Y.-P. Liu, G.C. Lynch, T.N. Truong, D.-H. Lu, D.G. Truhlar, J. Am. Chem. Soc. 115, 2408 (1993).

[31] A. Fernandez-Ramos and D.G. Thrular, J. Chem. Phys. 114, 1491 (2001).

[32] Y.-P. Liu, D.-J. Lu, A. Gonzalez-Lafont, D.G. Thrular, B.C. Garrett, J. Am. Chem. Soc. 115, 7806 
(1993).

[33] R. Meana-Panheda, D.G. Truhlar, and A. Fernandez-Ramos, J. Chem. Theory Comput. 6, 6 (2010)

[34] V.A. Benderskii, S.Yu. Grebenshchikov, D.E. Makarov, and E.V. Vetoshkin, Chem. Phys. 185, 101 (1994); V.A. Benderskii, E.V. Vetoshkin, E.I. Kats, and H.P. Trommsdorff, Phys. Rev. E 67, 026102 (2003); V.A. Benderskii, S.Yu. Grebenshchikov, E.V. Vetoshkin, G.V. Milnikov, and D.E. Makarov, J. Phys. Chem. 98, 3300 (1994); V.A. Benderskii, D.E. Makarov, and P.G. Grinevich, Chem. Phys. 170, 275 (1993).

[35] A. Fernández-Ramos, Z. Smedarchina, M.Z. Zgierski, W. Siebrand, J. Chem. Phys. 109, 1004 (1998); Z. Smedarchina, A. Fernández-Ramos, M.A. Rios, J. Chem. Phys. 106, 3956 (1997); Z. Smedarchina, M.Z. Zgierski, W. Siebrand, P.M. Kozlowski, J. Chem. Phys. 109, 1014 (1998); W. Siebrand, Z. Smedarchina, M.Z. Zgierski, A. Fernández- Ramos, Int. Rev. Phys. Chem. 18, 5 (1999); Z. Smedarchina, J. Comput. Chem. 22, 787 (2001).

[36] M. Kryvohuz, J. Chem. Phys. 134, 114103 (2001).

[37] S.C. Althorpe, J. Chem. Phys. 134, 114104 (2011); J.O. Richardson, S.C. Althorpe, and D.J. Wales, J. Chem. Phys. 135, 124109 (2011).

[38] W.H. Miller, Y. Zhao, M. Ceotto, and S. Yang, J. Chem. Phys. 119 (3), 1329 (2003).

[39] M. Ceotto and W.H. Miller, J. Chem. Phys. 120 (14), 6356 (2004).

[40] M. Ceotto, S. Yang, and W.H. Miller, J. Chem. Phys. 122, 044109 (2005).

[41] C. Venkataraman and W.H. Miller, J. Phys. Chem. A 108, 3035 (2004).

[42] T. Yamamoto and W.H. Miller, J. Chem. Phys. 120, 3086 (2004); T. Yamamoto and W.H. Miller, J. Chem. Phys. 122, 044106 (2005); Y. Zhao, T. Yamamoto and W.H. Miller, J. Chem. Phys. 120, 3100 (2004).

[43] W. Wang and Y. Zhao, J. Chem. Phys. 132, 064502 (2010); W. Wang, S. Feng, and Y. Zhao, J. Chem. Phys. 126, 114307 (2007); W. Wang and Y. Zhao, Phys. Chem. Chem. Phys. 13, 19362 (2011); W. Wang and Y. Zhao, J. Chem. Phys. 130, 114708 (2009).

[44] M. R. Buchowiecki and J. Vanicek., J. Chem. Phys. 132, 194106 (2010); T. Zimmermann and J. Vanicek, J. Mol. Model. 16 (11), 1779 (2010); T. Zimmermann and J. Vanicek, J. Chem. Phys. 131 (2), 024111 (2009); J. Vanicek and W.H. Miller, J. Chem. Phys. 127 (11), 114309 (2007); J. Vanicek, W.H. Miller, J.F. Castillo and F. Javier Aoiz, J. Chem. Phys. 123 (5), 054108 (2005).

[45] S. Yang and J. Cao, J. Chem. Phys. 122, 094108 (2005).

[46] A. Einstein, Dent. Ges. Berlin Verh. 19, No. 9/10 (1917); J. B. Keller, Ann. Phys. (N.Y.) 4, 180 (1950). 
[47] H. Jeffreys, Proc. Lond. Math. Soc. 23, 428 (1923); G. Wentzel, Z. Phys. 38, 518 (1926); H. A. Kramers, Z. Phys. 39, 828 (1926); L. Brollouin, Comptes Rendus de l'Academie des Sciences 183, 24 (1926).

[48] R.P. Feynman, Statistical Mechanics (Addison-Wesley, Reading, MA, 1972).

[49] T.M. Cherry, Philos. Trans. R. Soc. London, Ser. A 227, 651 (1928).

[50] R.J. Duchovic, Y.L. Volobuev, G.C. Lynch, A.W. Jasper, D.G. Trhular, T.C. Allison, A.F. Wagner, B.C. Garrett, J. Espinosa-Garcia, and J.C. Corchado, POTLIB Database at http://t1.chem.umn.edu/potlib.

[51] K.Takatsuka, H.Ushiyama, and A.Inoue-Ushiyama, Phys. Rep. 322, 347 (1999).

[52] E.M. Mortensen and K.S. Pitzer, Chem. Soc. (London) Spec. Publ. 16, 57 (1962).

[53] R.A. Marcus, J. Chem. Phys. 45, 4493 (1966).

[54] E.A. McCullogh and R.E. Wyatt, J. Chem. Phys. 54, 3578 (1971).

[55] T.F. George and W.H. Miller, J. Chem. Phys. 57, 2458 (1972).

[56] W.H. Press, B.P. Flannery, S.A. Teukolsky, W.T. Vetterling, Numerical Recipes in Fortran: The Art of Scientific Computing (Cambridge University Press, Cambridge, 1999)

[57] D.C. Clary, B.C. Garrett, and D.G. Truhlar, J. Chem. Phys. 78, 777 (1983).

[58] D.G. Truhlar and A. Kuppermann, J. Chem. Phys. 56, 2232 (1972) and D.G. Trhular, A. Kuppermann, and J.T. Adams, J. Chem. Phys. 59, 359 (1973).

[59] D.K. Bondi, J.N.L. Connor, B.C. Garrett, and D.G. Truhlar, J. Chem. Phys. 78, 5981 (1983).

[60] M.C. Gutzwiller, J. Math. Phys. 12, 343 (1971).

[61] E.T. Whittaker, A treatise on the Analytical Dynamics of Particle and Rigid Bodies (Cambridge University, Cambridge, 1965), pp.395-406. 


\section{Figure captions:}

Figure 1:Classical trajectories on the inverted potential of a symmetric (ABA type) collinear reaction: Red line and arrows represent the instanton trajectory; vertical dashed line the dividing surface, cross the transition state location. Initial $q_{2}$ values are 2.351 (green), 2.347 (instanton) and 2.344 (blue) trajectories for the $\mathrm{H}+\mathrm{BrH}$ potential in mass-scaled normal mode coordinate.

Figure 2: Value of the Hamilton action (continuos black line) and least action (dashed red line) respect to the initial trajectory position $\left(q_{2}\right)$ along the dividing surface $\left(q_{1}=0\right)$ for the collinear $H+H_{2}$ LSTH PES. A discontinuity of the first derivatives indicates the periodic trajectory conditions.

Figure 3: Monodromy matrix eigenvalues for initial positions and momenta in the case of collinear $\mathrm{H}+\mathrm{H}_{2}$ reaction. The line showed by the eigenvalues peaks determines the set of periodic trajectories initial conditions.

Figure 4: Phase space plot of a typical instanton trajectory. Left panel for the asymmetric stretch subspace and right panel for the symmetric one.

Figure 5: Instanton trajectories for four types of collinear reactions in Jacobi's coordinates. The transition state is located at the origin. The following color-code is employed to denote each temperature: At $T=100 \mathrm{~K}$, the trajectory is cyan, at $T=150 \mathrm{~K}$ red, at $T=175 \mathrm{~K}$ violet, at $T=$ $200 \mathrm{~K}$ green, at $T=225 \mathrm{~K}$ brown, at $T=250 \mathrm{~K}$ blue, at $T=275 \mathrm{~K}$ turquoise, at $T=300 \mathrm{~K}$ orange and at $T=350 \mathrm{~K}$ it is magenta.

Figure 6: Same as in Figure 5 but with bond coordinates reference system.

Figure 7: Variation of the potential $V\left(q_{1}(\tau), q_{2}(\tau)\right)$ along the instanton trajectory: Black continuos line for the $\mathrm{H}+\mathrm{BrH}$ reaction; red dotted line for the $\mathrm{H}+\mathrm{H}_{2}$ reaction; green dashed line for the $\mathrm{Cl}+\mathrm{HCl}$ reaction; cyan dot-dashed line for the $\mathrm{Cl}+\mathrm{DCl}$ reaction.

Figure 8: Instanton energy versus $\beta=1 / k_{B} T$, where $k_{B}$ is the Boltzmann constant. Red circles for the $\mathrm{H}+\mathrm{H}_{2}$ reaction; Green circles for the $\mathrm{Cl}+\mathrm{HCl}$ reaction; Cyan circles for the $\mathrm{Cl}+\mathrm{DCl}$ reaction; Black circles for the $\mathrm{H}+\mathrm{BrH}$ reaction; The dashed lines are Transition States energy for each reaction, i.e. the top of the potential barrier.

Figure 9: Rate constants for collinear reactions: black squares for the exact values, red circles the semiclassical instanton values and cyan diamonds the classical transition state theory values. 


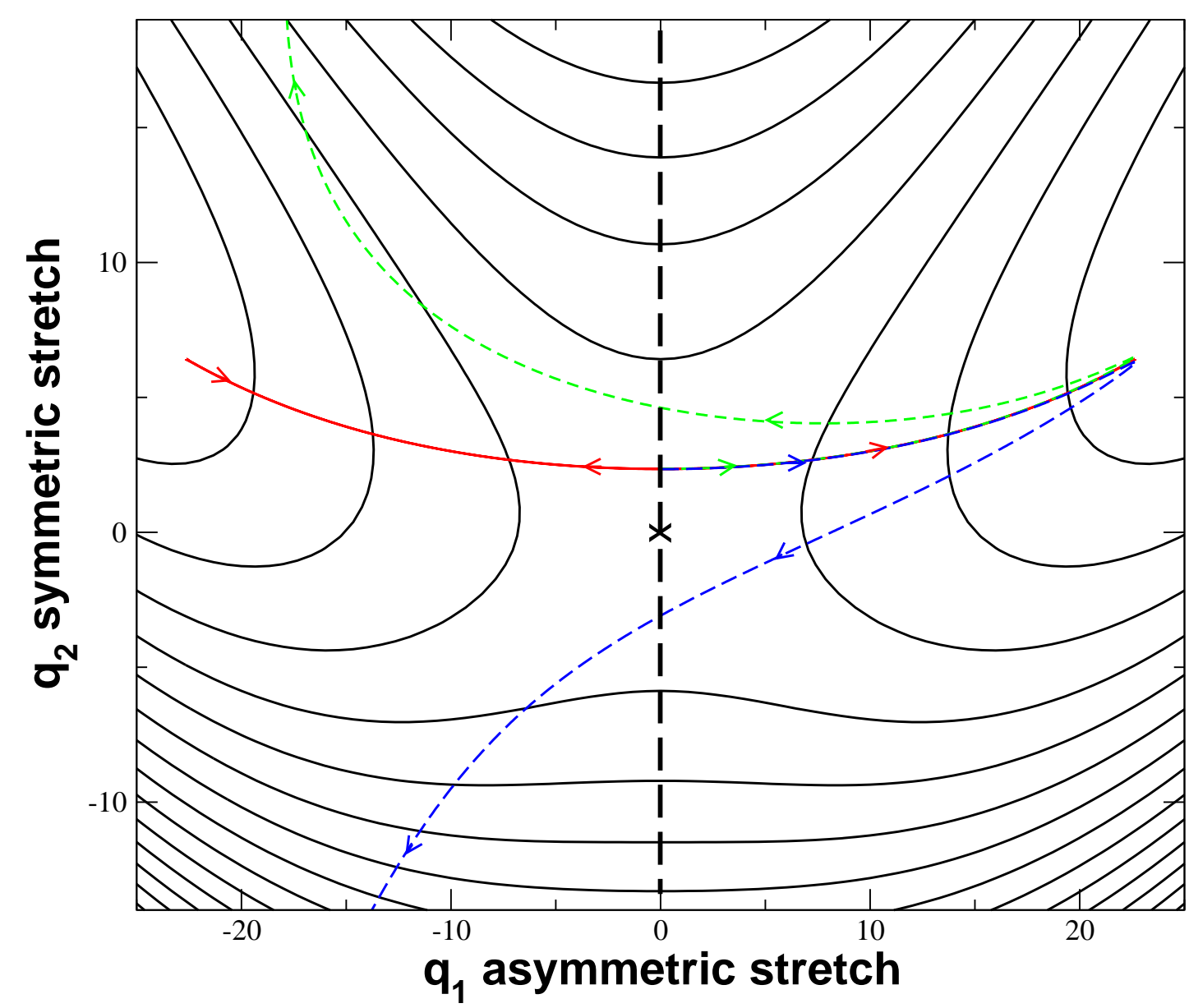




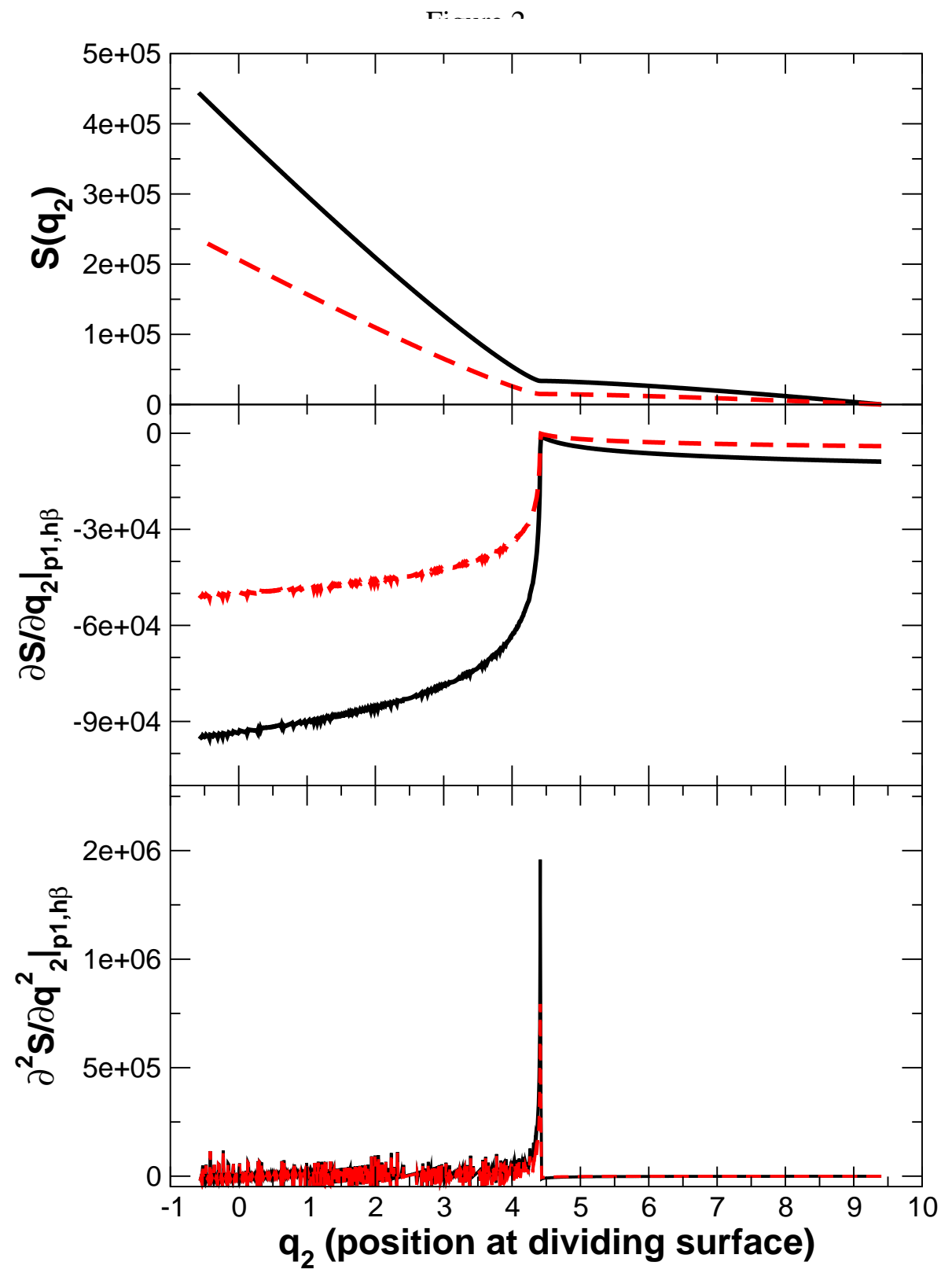


Figure3

eigenvalue

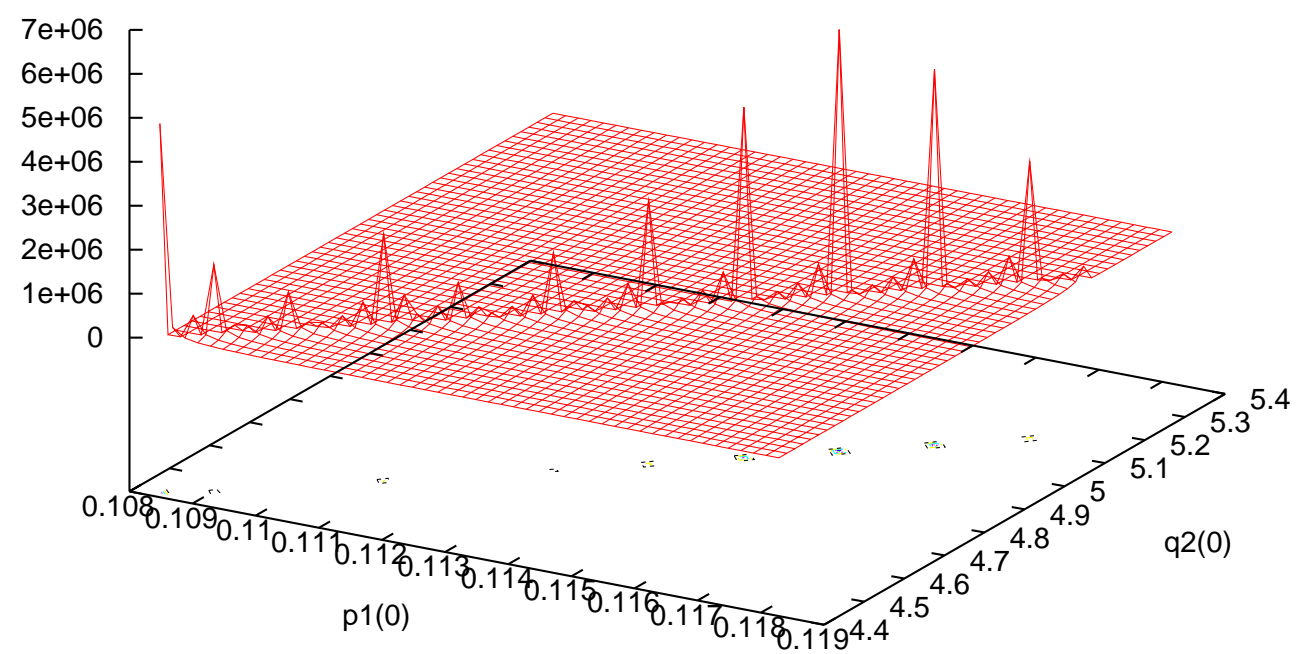


Figure 4
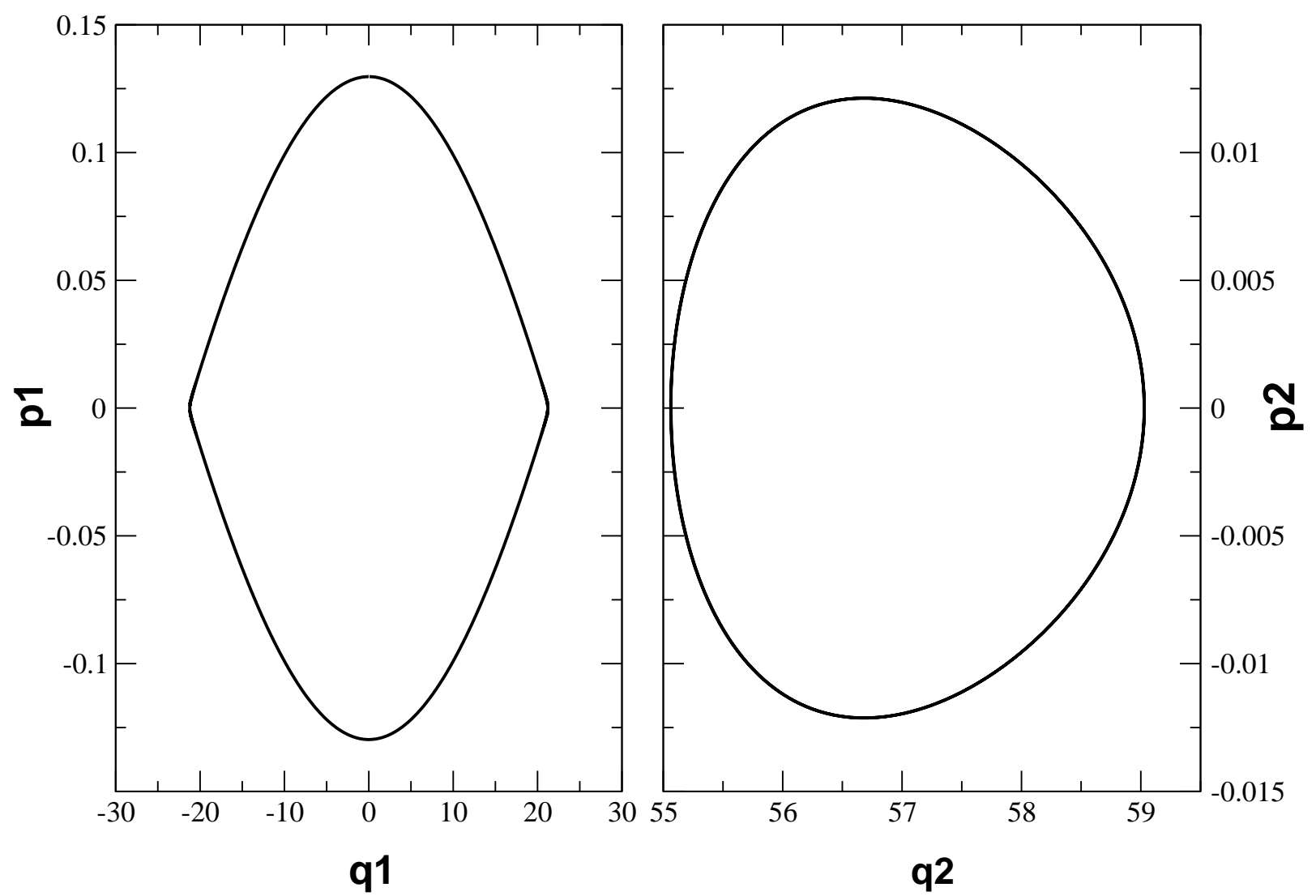

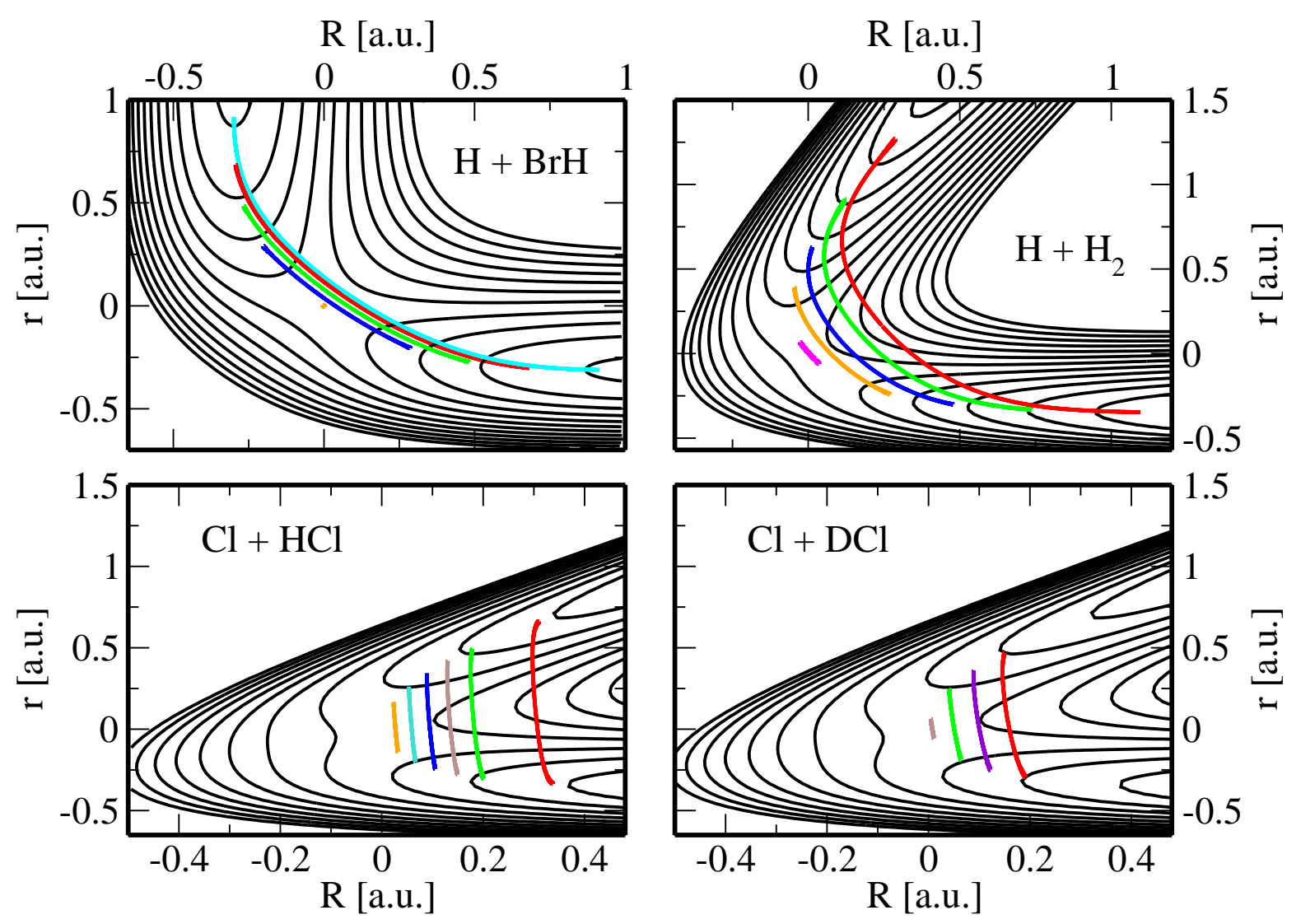

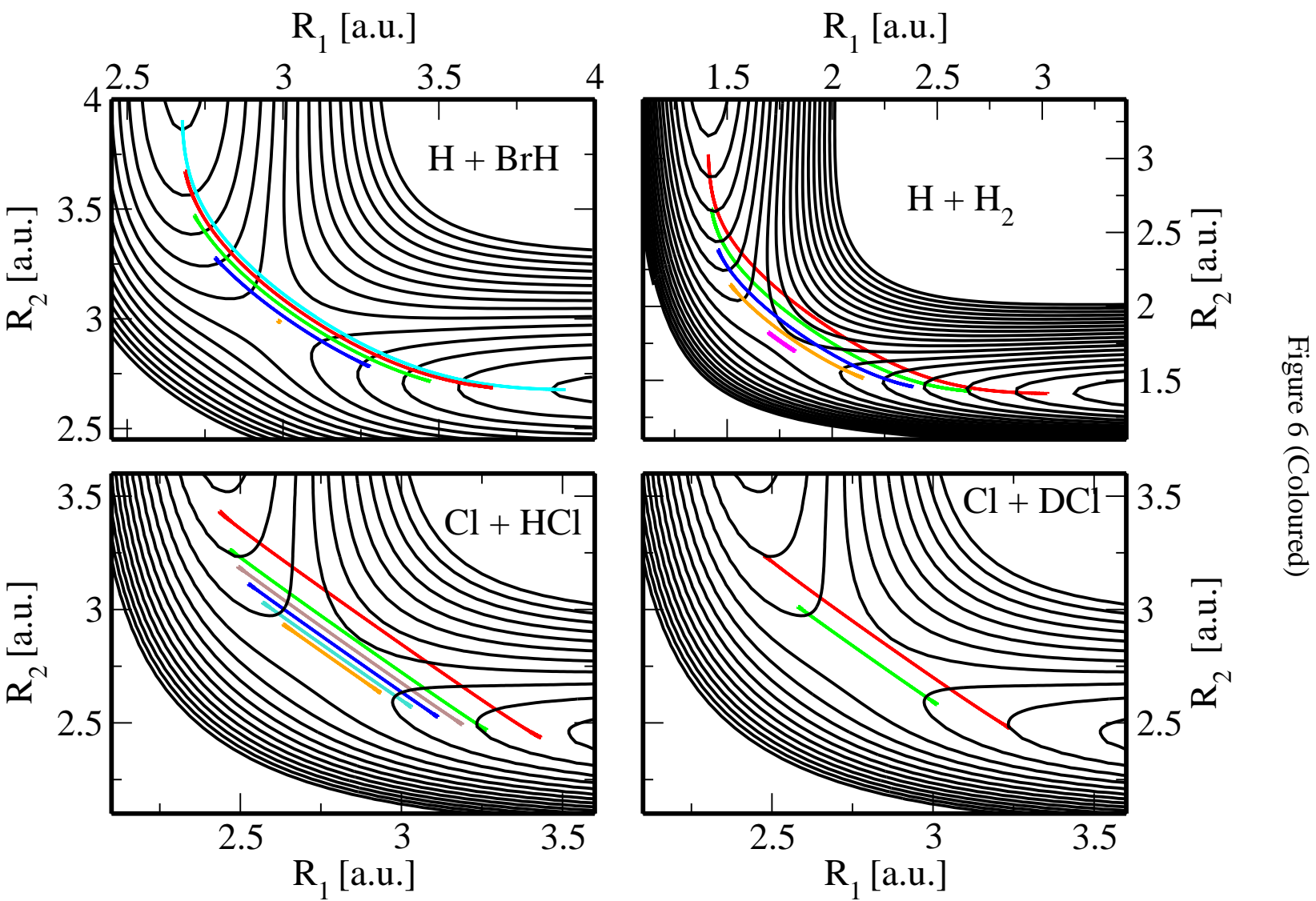


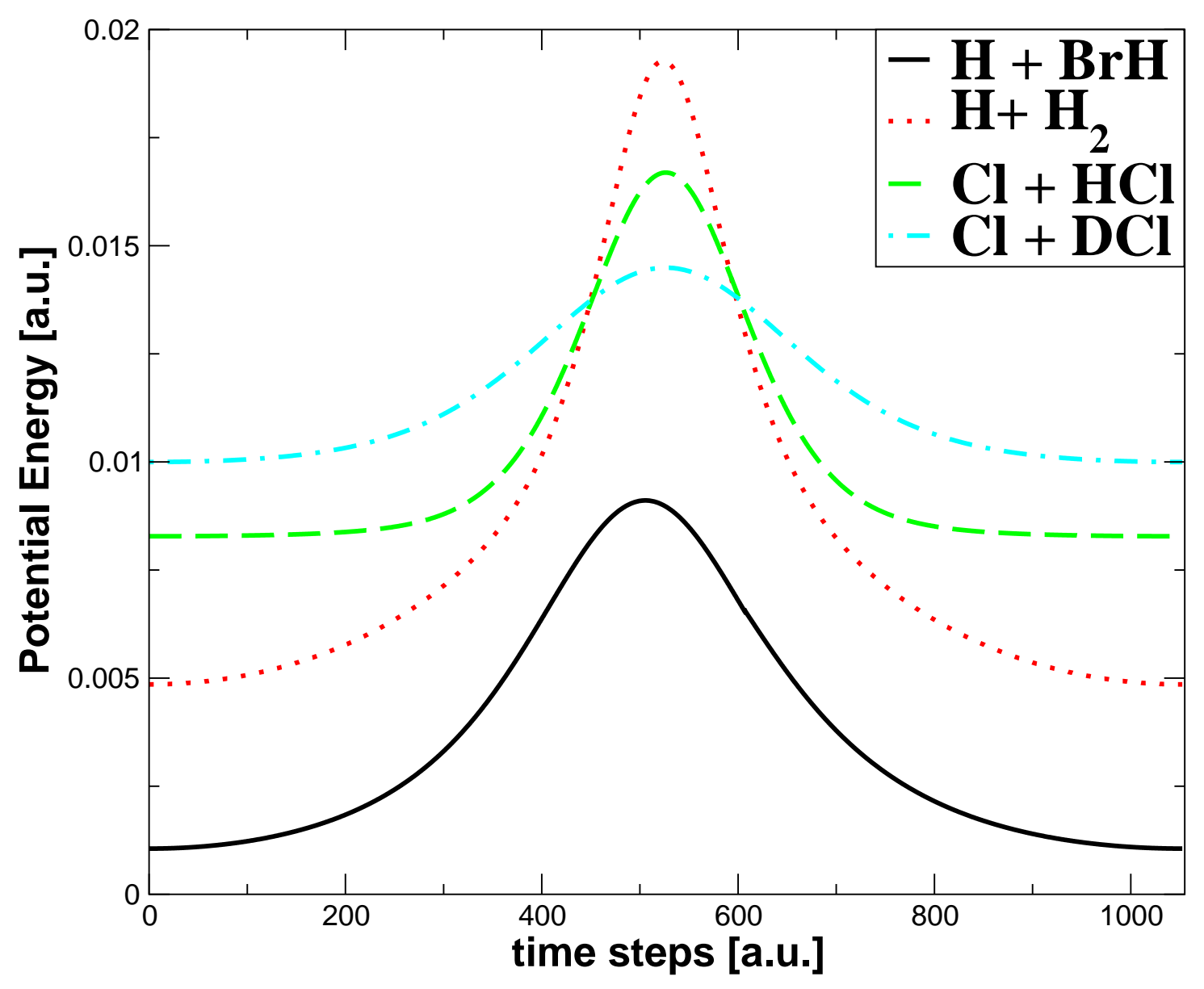




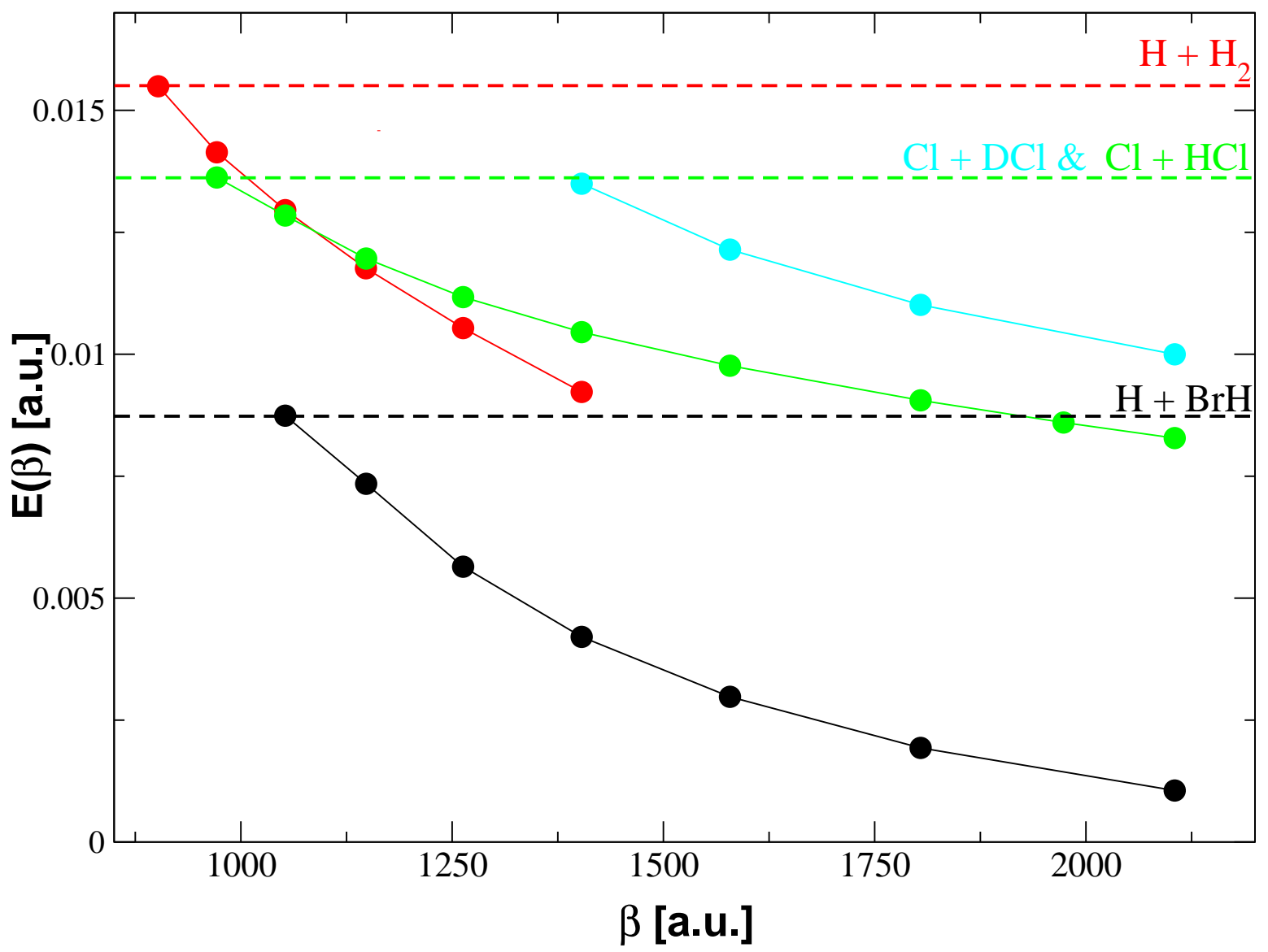



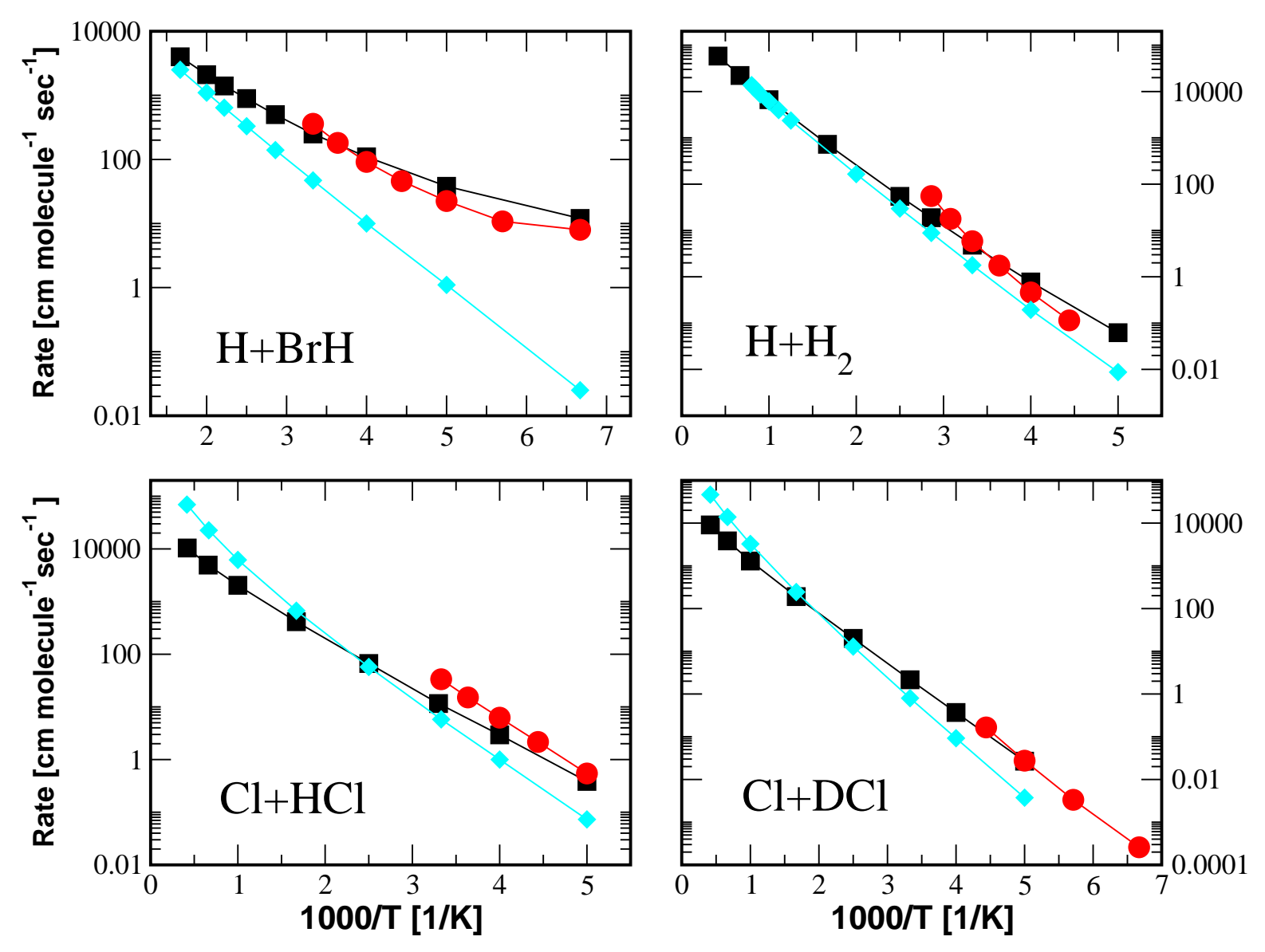

79
0
0
0
0 